Rev. Int. Contam. Ambie. 36 (4) 797-811, 2020

https://doi.org/10.20937/RICA.53318

\title{
MOVILIDAD DE ARSÉNICO EN LOS SEDIMENTOS DE UNA PRESA QUE RECIBE ESCURRIMIENTOS DE MINAS EPITERMALES
}

\author{
Arsenic mobility in the sediments of a dam that receives runoffs from epithermal mines \\ América BRAVO COVARRUBIAS ${ }^{1 *}$, Ester TORRES ${ }^{2}$, \\ Carlos AYORA ${ }^{2}$ y Yann René RAMOS ARROYO ${ }^{1}$
}

${ }^{1}$ Departamento de Hidráulica y Geomática, División de Ingenierías, Universidad de Guanajuato, Av. Juárez 77, Centro, Guanajuato.

${ }^{2}$ Consejo Superior de Investigaciones Científicas-Instituto de Diagnóstico Ambiental y Estudios del Agua (CSIC-IDAEA), Carrer Jordi Girona 18-26, Barcelona, España.

*Autora para correspondencia; americabc10@outlook.com

(Recibido: junio 2018; aceptado: agosto 2019)

Palabras clave: abastecimiento, extracción secuencial, transecto geoquímico, reservorio.

\section{RESUMEN}

Este trabajo tuvo como objetivo cuantificar $\mathrm{As}, \mathrm{Pb}, \mathrm{Fe}$ y $\mathrm{Mn}$ en las fases minerales de sedimentos recientes en una presa que recibe escurrimientos de minas abandonadas y plantear escenarios de retención y liberación de As en y desde el reservorio. La presa Santana (PS) se encuentra a la salida de una cuenca con tres embalses en cascada en el municipio de Guanajuato. Las cuatro muestras analizadas se recolectaron a $0.1 \mathrm{~m}$ de profundidad a lo largo de un transecto topográfico-geoquímico al interior de la PS. Se aplicó un protocolo de extracciones secuenciales de siete pasos considerando la reactividad y movilidad, para conocer de este modo los contenidos elementales en las distintas fases minerales y la potencial translocación entre ellas. Los extractos de cada fracción, así como una digestión ácida de cada muestra, se analizaron mediante masa de plasma acoplada inductivamente y emisión atómica plasmática acoplada inductivamente. Se generaron imágenes mediante microscopia electrónica de barrido de una muestra recolectada en la cortina. El contenido elemental total de sedimentos arrojó los siguientes valores (en $\mathrm{mg} \mathrm{kg}^{-1}$ ): As, 25.11 a $28.80 ; \mathrm{Pb}, 0.64$ a $2.83 ; \mathrm{Mn}, 562.3$ a 862.3; Fe, 51276.3 a 79 820.5. Desde la entrada y hacia la cortina, el As disminuye en la fracción de óxidos cristalinos; en contraste, se incrementa en la fracción intercambiable de óxidos amorfos y sulfuros. La concentración de Fe aumenta en la fracción intercambiable de óxidos amorfos y de sulfuros. El Mn se acumula cerca de la cortina, principalmente en fracciones de óxidos amorfos y cristalinos.

Key words: supply, sequential extraction, geochemical transect, reservoir.

\begin{abstract}
The objective of this work was to quantify $\mathrm{As}, \mathrm{Pb}, \mathrm{Fe}$ and $\mathrm{Mn}$ in the mineral phases of recent sediments in a dam that receives runoffs from abandoned mines and to propose scenarios of retention and release of As in and from the reservoir. The Santana dam (SD) is located at the exit of a basin with three reservoirs in cascade in the municipality
\end{abstract}


of Guanajuato. The four samples analyzed were collected at a depth of $0.1 \mathrm{~m}$ along a topographic-geochemical transect inside the SD. A protocol of sequential extractions of seven steps was applied considering the reactivity and mobility, in order to know the elemental contents in the different mineral phases and the potential translocations between them. The extracts of each fraction as well as an acid digestion of each sample were analyzed by inductively coupled plasma mass spectroscopy and inductively coupled plasma atomic emission. Images were generated by scanning electron microscopy of a sample collected in the curtain. The total elemental content in sediments yielded the following values (in $\mathrm{mg} \mathrm{kg}^{-1}$ ): As, 25.11 to 28.80; $\mathrm{Pb}, 0.64$ to 2.83; $\mathrm{Mn}, 562.3$ to 862.3; $\mathrm{Fe}, 51276.3$ to 79 820.5. From the entrance and towards the curtain, As decreases in the fraction of crystalline oxides; in contrast, it increases in the exchangeable fraction of amorphous oxides and sulphides. The concentration of iron increases in the exchangeable fraction of amorphous oxides and sulphides. Manganese accumulates near the curtain, mainly in the fractions of amorphous and crystalline oxides.

\section{INTRODUCCIÓN}

Los residuos mineros no controlados pueden afectar negativamente la calidad del agua superficial y subterránea, ya que aportan elementos potencialmente tóxicos (EPT) como $\mathrm{As}, \mathrm{Cd}, \mathrm{Cu}, \mathrm{Pb}, \mathrm{Tl}, \mathrm{Zn}$. Éstos se identifican por ser elementos que, presentes en altas concentraciones superiores a los valores de fondo, pueden provocar afectaciones al medio y la biota (Nordstrom 2011, Armiento et al. 2017). En la región fisiográfica de la Mesa Central de México, los yacimientos minerales más importantes son los de origen epitermal (Camprubí y Albinson 2006). Los yacimientos epitermales contienen oro y plata, y son producto de precipitados de una mezcla de aguas magmáticas y meteóricas emplazados a baja profundidad (menos de $1 \mathrm{~km}$ ) a temperaturas entre 150 y $350^{\circ} \mathrm{C}$. Comparados con otros tipos de yacimientos minerales, presentan enriquecimiento de elementos como Ag, As, Au, B, Hg S, Sb, Se, Te, Tl y U (Camprubí y Albinson 2006).

La principal fuente de EPT hacia los medios acuosos desde residuos mineros y minas epitermales proviene de la oxidación de sulfuros metálicos y de la disolución de hidroxisulfatos y sulfatos productos de este proceso (Plumlee 1999). Estos minerales se encuentran tanto en rocas con alteración hidrotermal como en los yacimientos y, sobre todo, en los residuos de la extracción expuestos a la atmósfera. La distribución de las especies de EPT en aguas naturales es función de varios factores. El más importante es el tipo de yacimiento, ya que éste condiciona la mineralogía y la intensidad de las interacciones agua-roca (Plumlee 1999).

A un reservorio de agua superficial, los EPT pueden ingresar asociados con materiales arrastrados por la escorrentía (sedimentos terrígenos) como partículas suspendidas o como especies disueltas (Smedley y Kinniburgh 2002). El agua almacenada es utilizada para el abasto a poblaciones y para las actividades productivas. En el primer caso deben cumplirse estándares mínimos de calidad de acuerdo a normas oficiales que regulan la salud de la población (SSA 1994).

Los reservorios favorecen la acumulación de sedimentos, los cuales son tanto fuente como sumideros de EPT, según las condiciones hidrodinámicas y geoquímicas al interior (Smedley et al. 2005, Chabukdhara y Nema 2012, Hansen 2012). En la matriz de los sedimentos, los metales existen en distintas especies químicas, las cuales condicionan las interacciones con el entorno, la movilidad, la disponibilidad biológica y el potencial tóxico.

Los sedimentos secundarios que se precipitan al interior de un reservorio que recibe escurrimientos de minas aportan información acerca del impacto en la calidad del recurso hídrico y ayudan a determinar el riesgo que se asocia con el almacenamiento de aguas de este tipo (Varol 2013). En México no existe una normatividad que regule el contenido de EPT en sedimentos de presas; sin embargo, pueden utilizarse como referencia los valores máximos de concentración de algunos países como Canadá y Estados Unidos, así como las normas europeas (CCME 2014, DWR 1995).

El conocimiento de la ocurrencia, movilidad y destino del As es un tema que tiene mucho interés. Se trata de un metaloide tóxico y con movilidad y especiación variable determinada por las condiciones fisicas y químicas del medio acuoso (Smedley y Kinniburgh 2002, Lee et al. 2015, Sharifi et al. 2016). El consumo de agua contaminada con este elemento se relaciona con afectaciones a la salud como cáncer, toxicidad cardiovascular y de hígado, 
y probablemente diabetes (Maull et al. 2012, Moon et al. 2012).

Un estudio realizado recientemente en la presa Santana (PS), ubicada en el estado de Guanjuato, México, se enfocó al análisis espacial y temporal de la química de la masa de aguas. Se recolectaron un total de 50 muestras en distintos puntos del embalse considerando su geometría durante cuatro campañas de muestreo en el periodo de diciembre de 2014 a noviembre de 2015. Los parámetros estudiados fueron: potencial de hidrógeno $(\mathrm{pH})$, temperatura $(\mathrm{T})$, oxígeno disuelto (OD), conductividad eléctrica $(\mathrm{CE})$, sulfatos $\left(\mathrm{SO}_{4}{ }^{2-}\right)$, sulfuros $\left(\mathrm{S}^{2-}\right)$, nitratos $\left(\mathrm{NO}_{3}{ }^{-}\right)$, cloruros $\left(\mathrm{Cl}^{-}\right)$, sodio $\left(\mathrm{Na}^{+}\right)$, potasio $\left(\mathrm{K}^{+}\right)$, calcio $\left(\mathrm{Ca}^{2+}\right)$, magnesio $\left(\mathrm{Mg}^{2+}\right)$, manganeso $\left(\mathrm{Mn}^{2+}\right)$, hierro $\left(\mathrm{Fe}^{2+}\right)$, arsénico total (Ast) y alcalinidad $\left(\mathrm{HCO}_{3}{ }^{-} \mathrm{y}\right.$ $\mathrm{CO}_{3}{ }^{2-}$ ) por volumetría. Las concentraciones máximas y mínimas de algunos componentes en la fase acuosa fueron: $\mathrm{pH}$ (7.46-10.02), CE (940-360 $\mu \mathrm{S} / \mathrm{cm})$; alcalinidad (1220-171 mg/L); sulfatos $(557-24 \mathrm{mg} / \mathrm{L})$; manganeso (2.1-0 mg/L); hierro (12.7-0 mg/L) y arsénico $(36-0 \mu \mathrm{g} / \mathrm{L})$ (Bravo-Covarrubias et al. 2017). Estos valores varían en función de la ubicación respecto de la cercanía a la cortina desde la entrada; también cambian sus valores con un gradiente de superficie a profundidad. Mediante el Código de modelación geoquímico PHREEQC2 (PH-potencial REdox-EQuilibrium, el cual está adaptado en $\mathrm{C}++$ ) (Parkhurst y Appelo 2013) se derivaron los índices de saturación de algunos minerales, prediciéndose en prácticamente todas las muestras la precipitación de ferrihidrita, pirita, calcita y dolomita (estos dos últimos deben su presencia a la geología del sitio).

Así como es importante el monitoreo de cuerpos de agua superficial, el estudio de la fracción sólida sedimentada proporciona información relevante acerca de la historia de acumulación e impactos potenciales a la calidad del agua. Las concentraciones elementales en sedimentos, las tasas de acumulación, así como las condiciones químicas que se desarrollan entre la columna de agua y los sedimentos, son factores que deben estudiarse para considerar posibles escenarios de impacto al recurso hídrico (Hansen 2012, Torres et al. 2013, Frémion et al. 2016). Una metodología que ha tenido amplia aplicación en el estudio de sedimentos es la de extracciones secuenciales (ES), muy útil para describir la movilidad de elementos desde una matriz sólida hacia fases acuosas cuando cambian las condiciones externas (Tessier et al. 1979). Existen varios procedimientos de ES (Tessier et al. 1979, Rauret et al. 1999, Dold, 2003, Torres y Auleda 2013) para extraer los EPT asociados con cada fase mineral $\mathrm{u}$ orgánica y cuantificarlos, con el fin de conocer su movilidad y toxicidad potencial. Evidentemente es necesaria una cuantificación total de los EPT en los sólidos, así como una caracterización mineralógica. El proceso de ES implica la utilización de diferentes soluciones a distintas condiciones que atacan secuencial y selectivamente los enlaces químicos en las fases minerales y la materia orgánica de las muestras sólidas (Rao et al. 2008). En cada paso del proceso se va incrementando la agresividad de la solución de extracción.

Los procedimientos de extracción secuencial (Tessier 1979, Kim et al. 2014, Aguilar-Hinojosa et al. 2016, Frémion et al. 2016) generalmente involucran las siguientes fracciones:

- Soluble en agua. Puede liberarse fácilmente en la masa de agua en eventos que favorezcan la disolución.

- Intercambiable catiónica o aniónica, según el caso. Esto se debe a la existencia de especies susceptibles a la variación por condiciones redox y de $\mathrm{pH}$, como en el caso del As (Wan et al. 2017).

- Asociada a carbonatos. Esta fracción podría movilizarse cuando descienda el $\mathrm{pH}$ del agua en contacto con los sedimentos. El As podría encontrarse asociado a calcita debido a procesos de coprecipitación.

- Fácilmente reducible. Contiene EPT asociados a oxihidróxidos de hierro y manganeso amorfos. Son compuestos de alto poder de adsorción e inestables en ausencia de oxígeno (Dzombak y Morel 1990).

- Asociada a óxidos de hierro cristalinos. Los metales presentes en esta fracción podrían movilizarse a la fase acuosa cuando el sedimento se encuentre bajo condiciones fuertemente reductoras (Bowell 1994, Smedley et al. 2005).

- Asociada a fases oxidables, como materia orgánica y sulfuros. En esta fracción se liberan los EPT cuando las condiciones son oxidantes.

- Residual, también conocida como fase litogénica. Son aquellos metales unidos a minerales primarios, los cuales están formando parte de sus estructuras cristalinas.

El objetivo de este trabajo es cuantificar los EPT en las fases minerales de sedimentos recientes de una presa que recibe escurrimientos de mina, así como plantear escenarios de retención y liberación de As en y desde el reservorio. 


\section{MATERIAL Y MÉTODOS}

\section{Área de estudio}

La presa Santana (PS) es un pequeño embalse ubicado al noreste de la ciudad de Guanajuato, al sur de la provincia fisiográfica de la Mesa del Centro, en la región hidrológica 12 Lerma-Santiago, subcuenca río Guanajuato-Silao (Fig. 1a). La PS representa la salida de un sistema de tres presas en cascada en la cuenca Esperanza-Soledad-Santana (CESS), donde cada una de las subcuencas se encuentra limitada por una cortina (Fig. 1b). Las presas Esperanza y Soledad abastecen al $40 \%$ de la población de la ciudad de Guanajuato. El área de captación del conjunto CESS es de $66.89 \mathrm{~km}^{2}$ y en temporada de lluvias es común que ambas presas derramen agua y aporten escurrimientos a la PS. En temporada de estiaje, la cuenca Santana, con un área de $21.29 \mathrm{~km}^{2}$, mantiene un flujo base constante que proviene tanto de manantiales como de las descargas continuas de tres minas abandonadas del sistema de vetas epitermales La Luz (Randall et al. 1994; Fig. 1b). La cuenca Santana tiene un gradiente de elevación de 2572 msnm a 1986 msnm (la cota de derrame de la PS).

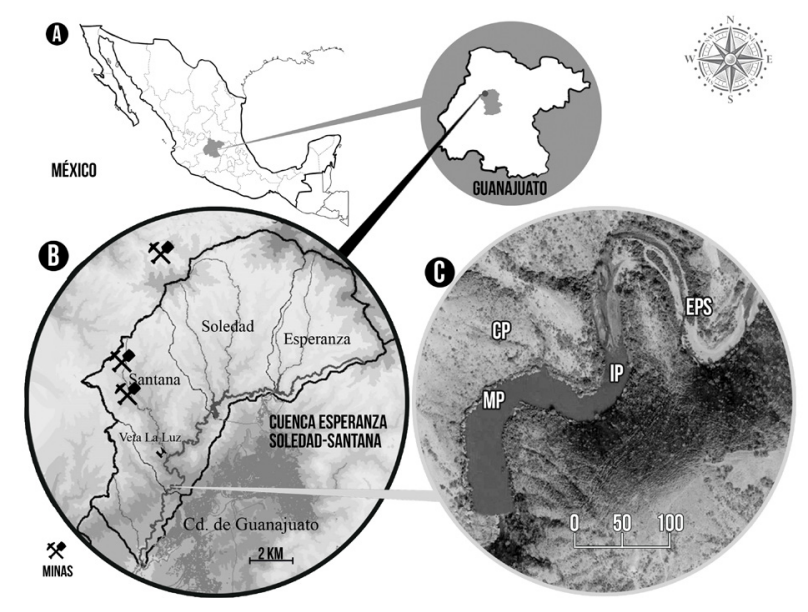

Fig. 1. (a) Ubicación de la cuenca al centro el estado de Guanajuato, (b) cuenca Esperanza-Soledad-Santana (los rombos negros representan las minas abandonadas en el sistema de vetas La Luz), (c) Presa Santana mostrando los cuatro puntos de recolección de sedimentos. Coordenadas de la cortina de la presa: $21^{\circ} 00^{\prime} 41^{\prime \prime} \mathrm{N}$, $101^{\circ} 17$ ' 50 "' O.

Se derivó la estructura de la PS por medio de una batimetría (Jofre-Meléndez et al. 2017), y se encontró que tiene una extensión lineal desde el arroyo de entrada hasta la cortina de $0.438 \mathrm{~km}$. El área que ocupa el embalse a su máxima capacidad a 1986 msnm es de $0.0178 \mathrm{~km}^{2}$. Originalmente, la PS tenía una capacidad de almacenamiento de $350000 \mathrm{~m}^{3}$, pero actualmente contiene $300000 \mathrm{~m}^{3}$ de azolves $\mathrm{y}$ sólo almacena $50000 \mathrm{~m}^{3}$ de agua. La profundidad máxima actual en la cortina es de $5 \mathrm{~m}$.

Las rocas que afloran en la CESS son ígneas intrusivas, diorita y tonalita del cretácico (Randall et al. 1994). Su porosidad es por fracturamiento y hay manantiales asociados. La composición de los fluidos hidrotermales favoreció el relleno de las fisuras con calcita y epidota, razón por la que el pH de los escurrimientos varía de 7.5 a 9, siendo las condiciones alcalinas (Ramos et al. 2010).

La principal fuente de solutos en la cuenca Santana son los minerales que rellenan las fracturas y sobre todo las vetas epitermales. Los minerales en la mena son electrum, argentita y aguilarita, polibasita, calcopirita, esfalerita y galena. La ganga en las vetas se compone de pirita, arsenopirita, calcita y cuarzo (Randall et al. 1994). El agua que escurre continuamente desde estas minas presenta concentraciones de arsénico de 20 a $350 \mu \mathrm{g} / \mathrm{L}$, manganeso de 0.2 a $4 \mathrm{mg} / \mathrm{L}$ y sulfatos de 500 a 4000 mg/L (Ramos et al. 2010).

La CESS tiene un régimen climático representativo de la Mesa Central, con una precipitación media anual de $790 \mathrm{~mm}$ y variaciones de lluvia anual de 230 a $1500 \mathrm{~mm}$. Esta condición de variabilidad climática es tanto temporal como espacial, pues hay un gradiente altitudinal de temperatura y precipitación. Las partes altas de la CESS tienen una temperatura media anual $6{ }^{\circ} \mathrm{C}$ más fría y presentan lluvias promedio de $850 \mathrm{~mm}$. La cuenca Santana recibe una precipitación anual promedio de $650 \mathrm{~mm}$ (Martínez-Arredondo et al. 2015).

\section{Muestreo}

La estrategia de muestreo consideró detectar patrones de movilidad física y química al interior de la presa. Las muestras se recolectaron en septiembre de 2015 a lo largo del eje principal de la presa utilizando una lancha. Los sedimentos recolectados y analizados (cuatro muestras) para este estudio son de reciente formación, de máximo $10 \mathrm{~cm}$ de profundidad y de naturaleza predominantemente química (de acuerdo con datos recabados en este estudio). La estrategia de muestreo consideró seleccionar dos ambientes contrastantes: oxidante y reductor, de acuerdo con los sitios de recolección que se muestran en el cuadro I y la figura 1c. Para la recolección se utilizó una draga que se lavó después de cada operación.

La importancia de la ubicación de las muestras radica en que el sitio de entrada de la presa Santana 
CUADRO I. UBICACIÓN DE LOS SITIOS DE MUESTREO EN LA PRESA SANTANA.

\begin{tabular}{lcccl}
\hline SITIO & X-UTM & Y-UTM & Profundidad $(\mathrm{m})$ & Importancia \\
\hline EPS & 14261441 & 2325322 & 0.3 & Textura gruesa, ambiente oxidante \\
IP & 14261339 & 2325366 & 1.5 & Inicio de la presa, ambiente oxidante \\
MP & 14261364 & 2325293 & 2.11 & Mitad de la presa, ambiente oxidante \\
CP & 14261204 & 2325121 & 4.95 & Cortina, procesos de reducción intensos \\
\hline
\end{tabular}

UTM: sistema de coordenadas Universal Transversal de Mercator; EPS: entrada presa Santana; IP: inicio de presa; MP: mitad de presa; CP: cortina de presa.

(EPS) proporciona información acerca de la naturaleza de los sedimentos antes de entrar a la presa. La muestra inicio de presa (IP) se recolectó al inicio del reservorio, en tanto que la muestra mitad de presa (MP) se encuentra en un ambiente aún bien oxigenado (a la mitad de la presa), y el sitio cortina de la presa $(\mathrm{CP})$ está cercano a la cortina, en un ambiente reductor debido a la materia orgánica, que se comporta como un consumidor de oxígeno presente en la masa de agua (Cuadro II).

Todos los reactivos utilizados fueron de grado analítico y las soluciones se prepararon con agua deionizada (Milli-Q). El material (plástico y de vidrio) utilizado para este estudio se lavó con $\mathrm{HNO}_{3}$ al $10 \%(\mathrm{v} / \mathrm{v})$ durante al menos $24 \mathrm{~h}$. Las muestras recolectadas se almacenaron en bolsas plásticas selladas y se mantuvieron a $4{ }^{\circ} \mathrm{C}$ para su posterior análisis en laboratorio (Torres y Auleda 2013).

\section{Extracciones secuenciales}

Las extracciones se realizaron por duplicado para asegurar una precisión analítica de este método. Las muestras se secaron a temperatura ambiente y posteriormente se sometieron a un protocolo modificado de acuerdon con Torres y Auleda (2013), consistente en siete pasos de extracción secuencial. En el cuadro III se resume la serie de pasos seguidos y sus condiciones experimentales. En un tubo Falcon se pusieron en contacto $0.2 \mathrm{~g}$ de sedimento con la fase extractante en constante agitación, y en algunos ca- sos calentamiento. Posteriormente se centrifugó el conjunto (sedimento-fase extractante) para separar la fase sólida y recolectar $1 \mathrm{~mL}$ de sobrenadante de cada una de las fracciones. El sobrenadante se acidificó con $10 \mathrm{~mL}$ de ácido nítrico al $2 \%$ y posteriormente se realizó el análisis de elementos mayoritarios y traza. Las concentraciones de $\mathrm{Fe}, \mathrm{Ca}, \mathrm{Mg}, \mathrm{Na}, \mathrm{K}$ y S en los sedimentos se midieron por emisión atómica plasmática acoplada inductivamente (ICP-AES, por sus siglas en inglés) con un equipo Thermo JarrelAsh y un CID detector con límites de detección de $0.2 \mathrm{mg} / \mathrm{L}$ para Fe y $0.5 \mathrm{mg} / \mathrm{L}$ para $\mathrm{S}$. Los metales traza se determinaron por la masa de plasma acoplada inductivamente (ICP-MS, por sus siglas en inglés) con un detector CID con límites de detección para estos elementos del orden de $1 \mu \mathrm{g} / \mathrm{L}$. Todas las técnicas analíticas fueron calibradas con una serie de estándares, y los coeficientes de regresión lineal fueron mayores a 0.999 .

Se realizó una segunda fase extractante en la fase sólida restante, cumpliendo con los requerimientos de cada paso, y así sucesivamente hasta completar el número de pasos del protocolo.

Para realizar el proceso de digestión total se utilizaron $0.1 \mathrm{~g}$ de sedimento seco; éstos se depositaron en un reactor de teflón, al cual se agergaron $10 \mathrm{~mL}$ de $\mathrm{HNO}_{3}$ concentrado y $5 \mathrm{~mL}$ de $\mathrm{HClO}_{4}$ concentrado. La mezcla se calientó a $135^{\circ} \mathrm{C}$ en un sistema de horno de grafito hasta llegar a sequedad. Se dejó enfriar y se le adaptó un codo a cada reactor. Nuevamente se

CUADRO II. PROPIEDADES DE LOS SEDIMENTOS ANALIZADOS.

\begin{tabular}{llllcc}
\hline Muestra & Característica & Textura & Color & $\begin{array}{c}\mathrm{CaCO}_{3} \\
(\%)\end{array}$ & $\begin{array}{c}\text { Materia orgánica } \\
(\%)\end{array}$ \\
\hline EPS & Antes de la presa & Arena & Café & $0-5$ & 1.63 \\
IP & Inicio de la presa & Arena & Café & $5-10$ & 2.73 \\
MP & Ambiente oxidante & Limo-arcillosa & Negro & $10-15$ & 4.50 \\
CP & Ambiente reductor & Limo-arcillosa & Café oscuro & $10-15$ & 9.81 \\
\hline
\end{tabular}

EPS = entrada presa EPS: entrada presa Santana; IP: inicio de presa; MP: mitad de presa; CP: cortina de presa. 
CUADRO III. PROTOCOLO DE EXTRACCIÓN SECUENCIAL APLICADO.

\begin{tabular}{|c|c|c|c|c|}
\hline Fracciones & Fase extractante & Tiempo de contacto & $\begin{array}{l}\text { Condiciones } \\
\text { Experimentales }\end{array}$ & $\begin{array}{l}\text { Posibles fases que se } \\
\text { atacan }\end{array}$ \\
\hline $\begin{array}{l}\text { Fracción soluble } \\
\text { en agua }\end{array}$ & Agua MilliQ (50 mL) & $1 \mathrm{~h}$ & Temperatura ambiente & Sulfatos secundarios \\
\hline $\begin{array}{l}\text { Fracción } \\
\text { carbonatos }\end{array}$ & $\begin{array}{l}\text { Acetato- } \mathrm{NH}_{4} 1 \mathrm{M} \\
\mathrm{pH} 4.5(20 \mathrm{~mL})\end{array}$ & $2 \mathrm{~h}$ & Temperatura ambiente & $\begin{array}{l}\text { Contenido de calcita } \\
\text { Iones adsorbidos e } \\
\text { intercambiables }\end{array}$ \\
\hline $\begin{array}{l}\mathrm{Fe}(\mathrm{III}) \\
\text { oxihidróxidos }\end{array}$ & $\begin{array}{l}\text { Oxalato- } \mathrm{NH}_{4} 0.2 \mathrm{M}+ \\
\text { acido oxálico } 0.2 \mathrm{M} \\
\text { pH } 3(20 \mathrm{~mL})\end{array}$ & $1 \mathrm{~h}$ & $\begin{array}{l}\text { Temperatura ambiente } \\
\text { Ausencia de luz }\end{array}$ & $\begin{array}{l}\text { Schwertmannite } \\
\text { Ferridrita } \\
\text { Jarosita secundaria y } \\
\mathrm{MnO}_{2}\end{array}$ \\
\hline $\begin{array}{l}\text { Fe(III) } \\
\text { óxidos }\end{array}$ & $\begin{array}{l}\text { Oxalato- } \mathrm{NH}_{4} 0.2 \mathrm{M}+ \\
\text { acido oxálico } 0.2 \mathrm{M} \\
\text { pH } 3(20 \mathrm{~mL})\end{array}$ & $1 \mathrm{~h}$ & Temperatura $80^{\circ} \mathrm{C}$ & $\begin{array}{l}\text { Goetita } \\
\text { Jarosita } \\
\text { Hematita } \\
\text { Magnetita } \\
\text { Ferridrita de orden elevado }\end{array}$ \\
\hline $\begin{array}{l}\text { Fracción } \\
\text { orgánica }\end{array}$ & $\mathrm{NaOH} 0.5 \mathrm{M}$ & $16 \mathrm{~h}$ & Temperatura ambiente & $\begin{array}{l}\text { Contenido de materia } \\
\text { orgánica }\end{array}$ \\
\hline $\begin{array}{l}\text { Fracción resto } \\
\text { de sulfuros }\end{array}$ & $\mathrm{HNO}_{3} 8 \mathrm{M}(20 \mathrm{~mL})$ & $3 \mathrm{~h}$ & Temperatura $80^{\circ} \mathrm{C}$ & $\begin{array}{l}\text { Contenido de S-elemental } \\
\text { y } \\
\text { S-pirítico }\end{array}$ \\
\hline $\begin{array}{l}\text { Fase } \\
\text { residual }\end{array}$ & $\begin{array}{l}\mathrm{HNO}_{3} \text { al } 65 \% \\
\mathrm{HClO}_{4} \text { (concentrado) } \\
(5 \mathrm{~mL}+5 \mathrm{~mL})\end{array}$ & $16 \mathrm{~h}$ & Temperatura $135^{\circ} \mathrm{C}$ & Minerales residuales \\
\hline
\end{tabular}

calienta a $135^{\circ} \mathrm{C}$ hasta que los ácidos se evaporaron por completo y se condensaron en los codos. En seguida se agregaron $3 \mathrm{~mL}$ de $\mathrm{HNO}_{3}$ concentrado y la mezcla se calientó a $135^{\circ} \mathrm{C}$ para disolver las sales. Finalmente, la solución se filtró a través de jeringas con filtro de nylon de $0.22 \mu \mathrm{m}$, se diluyó a $10 \mathrm{~mL}$ y se almacenó a $4{ }^{\circ} \mathrm{C}$ hasta su análisis mediante ICP.

\section{Obtención de micrografías de las muestras de sedimento}

Se realizó una serie de análisis por microscopia electrónica de barrido (SEM, por sus siglas en inglés) con un microscopio modelo JEOL-JMS-5410 en la muestra $\mathrm{CP}$, con la finalidad de validar los datos e información de los resultados analíticos de las extracciones secuenciales, además de que dicho proceso aporta un análisis semicuantitativo acerca de la composición elemental de los minerales estudiados. La muestra fue homogeneizada y se preparó colocando el sedimento seco sobre un porta muestras, para posteriormente recubrirla con una fina película de carbono.

El microscopio electrónico de barrido utilizado está equipado con dos diferentes detectores. El detec- tor para generar imágenes con electrones secundarios (SEI, por sus siglas en inglés) permite apreciar la muestra con alta resolución, mientras que la captada con el detector para imágenes de electrones retrodispersados (BEI, por sus siglas en inglés) permite la obtención de imágenes de composición y topografía de la superficie.

\section{RESULTADOS Y DISCUSIÓN}

\section{Condiciones físicas de la PS}

Este reservorio no se utiliza en la actualidad para abastecer a la población de la ciudad de Guanajuato. Tiene una antigüedad de 230 años y fue construido para la industria minera que operaba en ese tiempo. El volumen actual de almacenamiento es de $50000 \mathrm{~m}^{3}$ y su volumen total $\left(350000 \mathrm{~m}^{3}\right)$ ha disminuido por la cantidad de sedimentos que se van acumulando, tanto los terrígenos como los que han precipitado al interior. Considerando la edad y la pérdida de volumen de captación $\left(300000 \mathrm{~m}^{3}\right)$ puede derivarse una tasa de acumulación de sedimentos de $1.30410^{6} \mathrm{~kg}$ anuales. Considerando una densidad entre 1500 a $2000 \mathrm{~kg} / \mathrm{m}^{3}$ 
y el área de la presa $\left(17800 \mathrm{~m}^{2}\right)$, se deduce que anualmente la columna de sedimentos crece de 0.05 a $0.07 \mathrm{~m}$ (Bravo-Covarrubias et al. 2017).

Se puede considerar a los sedimentos como un depósito en potencia de EPT, los cuales son liberados a la columna de agua cuando cambian las condiciones físicas y químicas del medio donde se encuentran. Existe una conocida asociación de As en sedimentos con los óxidos metálicos, particularmente aquellos de Fe y Mn, razón por la cual en el presente estudio se analiza el comportamiento de dichos elementos. En el cuadro II se muestran algunas propiedades físicas de los sedimentos y de contenidos de carbonatos y materia orgánica. La matriz de los sedimentos sobre la cual se realizó el análisis presentó distintos contenidos elementales totales (valores en $\mathrm{mg} / \mathrm{kg}$ para As de 25.11 a 28.80, $\mathrm{Pb}$ de 0.64 a 2.83 , Mn de 562.3 a 862.3 y Fe de 51 276.3 a 79 820.5). A nivel macroscópico también fue posible observar una clara variación: mientras que a la entrada de la presa los sedimentos tienen grano grueso (grava y arena), en la parte cercana a la cortina es evidente una textura más fina, predominantemente de limo y arcilla. Con la disminución de tamaño de partícula al acercarse a la cortina se asocia un incremento del porcentaje de carbonatos y materia orgánica. Debido a que estos compuestos se precipitan, las condiciones del fondo de la presa son reductoras por la ausencia o disminución de oxígeno como resultado del consumo por la materia orgánica, y favorecen la acumulación de sedimentos químicos, tales como sulfuros metálicos.

El vaso de captación se encuentra sujeto a dinámicas hidrológicas que determinan tanto la tasa de acumulación de sedimentos como su potencial migración y/o acumulación. La PS recibe agua todo el año. Durante la temporada de secas (de noviembre a mayo) entra al vaso de captación un alto porcentaje de escurrimientos de aguas de mina, de aproximadamente $60 \%$ (Martínez-Arredondo et al. 2015). El flujo en temporada de estiaje (ausencia de lluvias) puede ser de $10 \mathrm{~L} / \mathrm{s}$, que equivale a $864 \mathrm{~m}^{3}$ (variación de 500 a $1000 \mathrm{~m}^{3}$ ) al día. Sin embargo, dado el volumen tan pequeño de la PS, en temporada de lluvias pueden presentarse escurrimientos máximos que desplazan todo el volumen de la presa en tiempos cortos como 2 o 3 h (Bravo-Covarrubias et al. 2017). Se ha cuantificado que pueden entrar hasta $500000 \mathrm{~m}^{3}$ al día por el canal principal durante la temporada de lluvias (Martínez-Arredondo et al. 2015). Es posible que la corriente llegue a socavar los sedimentos depositados en la profundidad y muy probablemente los movilice fuera del reservorio.

\section{Movilidad de los elementos en la matriz del se- dimento}

Las condiciones físicas y químicas, $\mathrm{pH}, \mathrm{T}, \mathrm{OD}$, $\mathrm{CE}, \mathrm{SO}_{4}{ }^{2-}, \mathrm{S}^{2-}, \mathrm{NO}_{3}{ }^{-}, \mathrm{Cl}^{-}, \mathrm{Na}^{+}, \mathrm{K}^{+}, \mathrm{Ca}^{2+}, \mathrm{Mg}^{2+}$, $\mathrm{Mn}^{2+}, \mathrm{Fe}^{2+}$, As y alcalinidad influyen en las reacciones químicas entre las especies minerales en la matriz de los sedimentos. Los gráficos mostrados describen un transecto que va de la entrada de la presa hacia la cortina. Para el arsénico (Fig. 2) se observa que la concentración total es muy similar para todos los sitios: en promedio de $25.25 \mathrm{mg} / \mathrm{kg}$. La fracción de As en óxidos cristalinos es predominante para los sitios EPS $(25.11 \mathrm{mg} / \mathrm{kg})$ e IP $(23.98 \mathrm{mg} / \mathrm{kg})$; sin embargo, esta condición cambia con la distancia desde la entrada para después ser mayoritaria en la fracción de óxidos amorfos (MP [23.10 mg/kg] y CP $[28.8 \mathrm{mg} / \mathrm{kg}])$. Este comportamiento sugiere que el As entra a la presa en forma particulada, adsorbido a óxidos de hierro y al parecer por arrastre como carga de fondo. Por las condiciones oxidantes y alcalinas, se sugiere que la especie de As predominante tanto en fase acuosa como enlazada a los óxidos es $\mathrm{HAsO}_{4}{ }^{2-}$. La figura 3 muestra los límites de estabilidad para especies acuosas y sólidas de As, Fe y S considerando sus estados de oxidación; se construyó adaptando el software MEDUSA, el cual se ha modificado (Puigdomenech et al. 2014) con las condiciones registradas en la PS de $\mathrm{pH}$, potencial redox y concentraciones máximas de As total, ferroso y sulfuros (Bravo-Covarrubias et al. 2017). En la figura 3 se muestra el cambio de condiciones en las zonas de la presa, desde la EPS hasta la CP. Este cambio de condiciones redox puede encontrarse en la columna de agua, desde la superficie hasta el fondo de la PS. Estas condiciones, además de favorecer la reducción de As (V) a As (III), también propician el cambio de

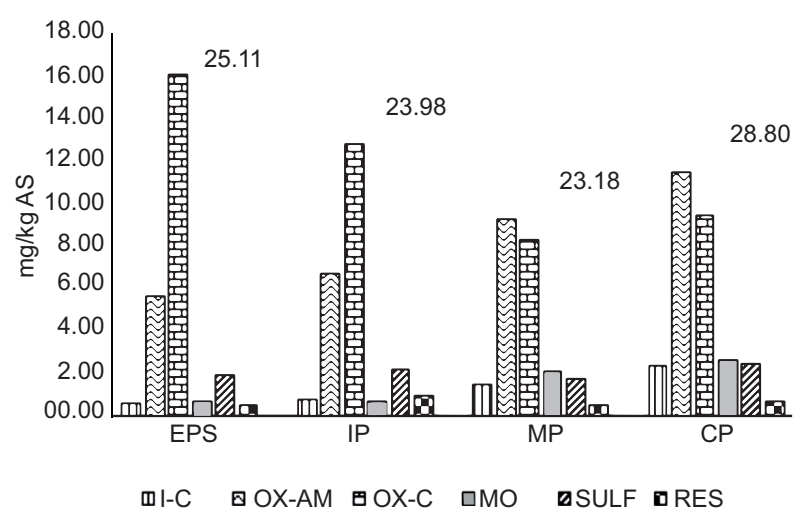

Fig. 2. Fracciones de la extracción secuencial para el elemento As. EPS: entrada presa Santana; IP: inicio de presa; MP: mitad de presa; CP: cortina de presa. 


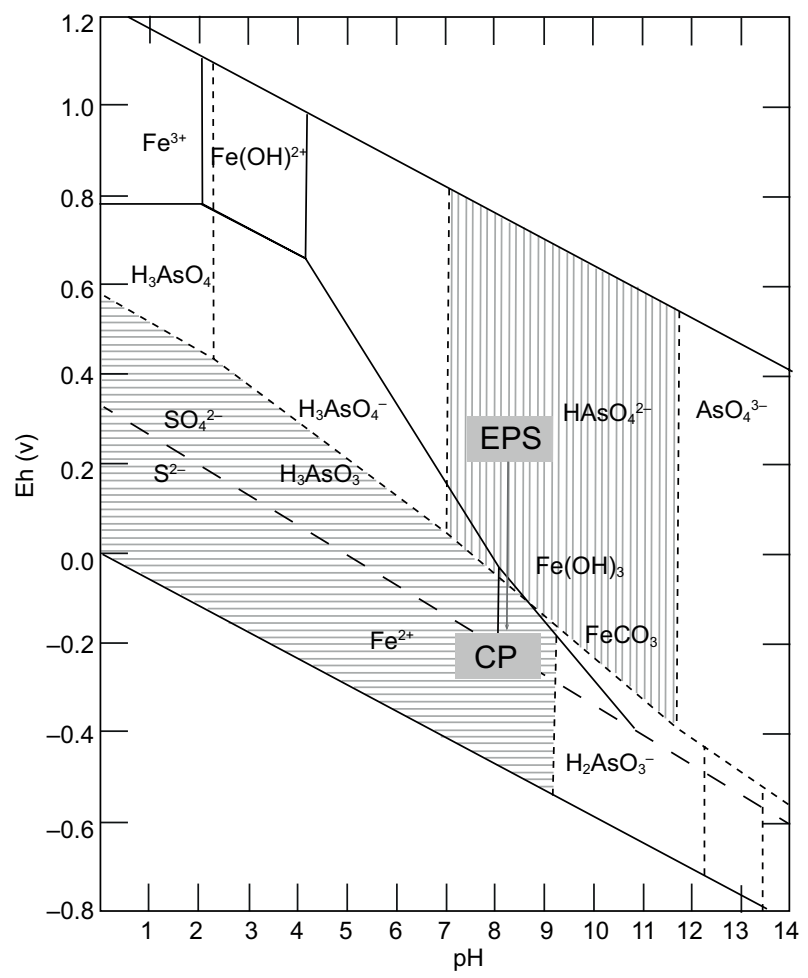

Fig. 3. Diagrama de estabilidad de especies de Fe, As y S mostrando la evolución del sitio entrada presa Santana (EPS) a cortina presa (CP). Gráfico elaborado con el programa MEDUSA utilizando las concentraciones para $\mathrm{Fe}^{2+}$ de $12.7 \mathrm{mg} / \mathrm{L}$, As de $0.034 \mathrm{mg} / \mathrm{L} \mathrm{y} \mathrm{S}^{2-}$ de $0.980 \mathrm{mg} / \mathrm{L}$, las máximas encontradas en la presa (Bravo et al. 2017).

$\mathrm{Fe}$ (III) a Fe (II) y de sulfatos a sulfuros, que controlan los procesos de adsorción, precipitación y coprecipitación de As. La zona de líneas verticales es donde predomina la especie $\mathrm{HAsO}_{4}^{-}$, que se encuentra en la columna de agua tanto disuelta como asociada a partículas suspendidas (menos tóxica) y la zona de líneas horizontales es donde predomina la especie $\mathrm{H}_{3} \mathrm{AsO}_{3}$ (más tóxica).

En la figura 4 se aprecia la evolución de las fracciones de As intercambiable-carbonatos, enlazadas a óxidos y como sulfuros a lo largo del transecto y expresadas en porcentajes. Se sugiere que hay un cambio en las especies de As tanto en la columna de agua como en las aguas de poro del sedimento de $\mathrm{HAsO}_{4}{ }^{2-}$ a las reducidas $\mathrm{H}_{2} \mathrm{AsO}_{3}{ }^{-} \mathrm{y} \mathrm{H}_{3} \mathrm{AsO}_{3}{ }^{0}$ según el $\mathrm{pH}$, cuya variación al interior de la presa es de $7.46 \mathrm{a}$ 10.02, detectándose los mayores valores en el fondo (Bravo-Covarrubias et al. 2015). Es posible distinguir que el contenido de As en la fracción intercambiablecarbonatos (I-C) aumenta a lo largo del transecto, siendo de $2.32 \%$ en el sitio EPS, $3.15 \%$ en IP, $6.47 \%$ en MP y $8.21 \%$ en CP. Dada la condición de que

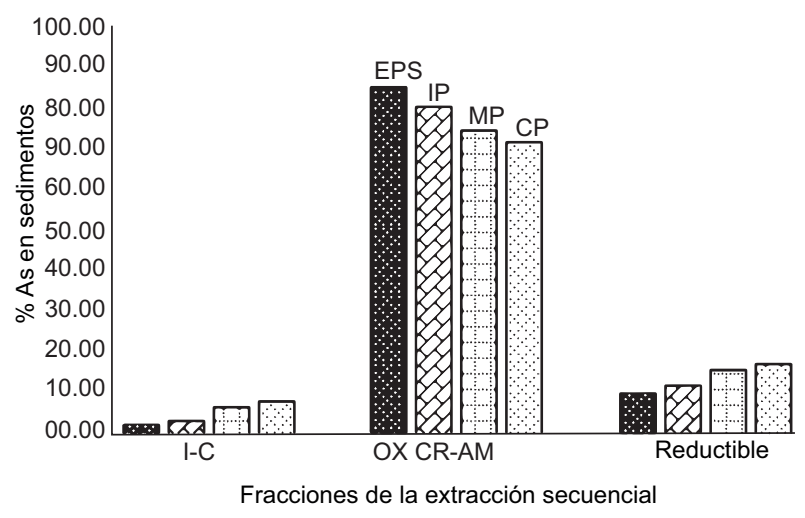

Fig. 4. Contenido porcentual de As en las fracciones intercambiable, óxidos cristalinos más amorfos y sulfuros: I-C: Intercambiable, OX CR-AM: óxidos cristalinos y amorfos, Reductible: reducible. EPS: entrada presa Santana, IP: inicio presa, MP: mitad presa, CP: cortina presa.

las aguas son alcalinas y existe la evidencia de que hay una saturación de calcita (Bravo-Covarrubias et al. 2017), se plantea que está ocurriendo una coprecipitación, inclusive una potencial adsorción como mencionan Romero et al. (2004). En la fase reducible (óxidos amorfos y cristalinos) tiende a disminuir en función del recorrido del transecto, yendo de un porcentaje mayor a menor de As: $\mathrm{EPS}=85.87 \%$, IP $=80.98 \%, \mathrm{MP}=75.19 \%, \mathrm{CP}=72.32 \%$. Mientras tanto, en la fracción de sulfuros el As se presenta en mayor porcentaje a lo largo del reservorio: EPS = $9.9 \%, \mathrm{IP}=11.9 \%, \mathrm{MP}=16.17 \%, \mathrm{CP}=17.24 \%$. Esto marca la pauta para concluir que el As se está movilizando de una fracción de óxidos hacia la fase de sulfuros.

Con el elemento hierro se observa una tendencia de acumulación a lo largo del gradiente, siendo mayor el contenido total en la cortina $(7.98 \%$ ) que en la entrada a la presa (5.12\%), como se aprecia en la figura 5. Para la fracción I-C se observa un aumento de la cantidad de hierro asociado a carbonatos, debido a la precipitación de siderita $\left(\mathrm{FeCO}_{3}\right)$, favorecida por el ambiente alcalino y reductor. A la entrada de la presa este porcentaje es de $1.7 \%$ y en la cortina de $3.3 \%$, prácticamente el doble. Visualizando el comportamiento de las especies de hierro, tanto en fase acuosa como mineral a lo largo del transecto de EPS a CP (Fig. 3), se plantea un cambio de $\mathrm{Fe}^{3+}$ en los oxihidróxidos y óxidos a $\mathrm{Fe}^{2+}$ tanto en siderita como en hierro, en ambos como sulfuros. De forma similar, el $\mathrm{Fe}$ aumenta en la fracción de óxidos amorfos (EPS $<$ IP $<$ MP $<\mathrm{CP}$ ); sin embargo, esta tendencia en términos de porcentajes se mantiene 


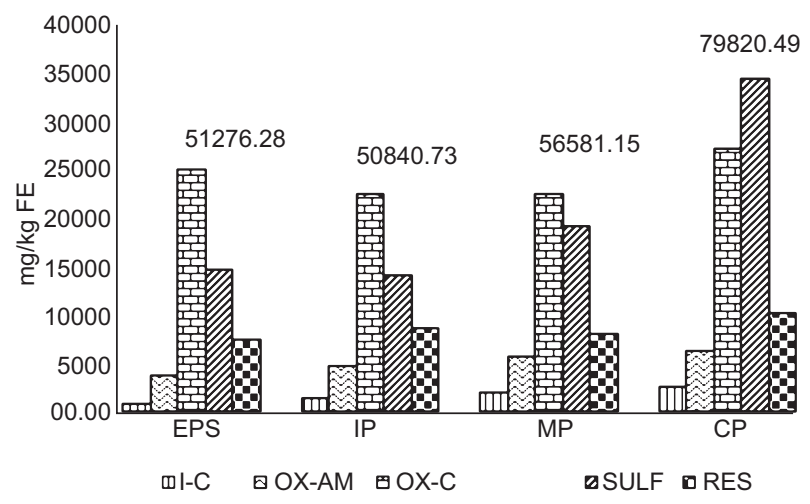

Fig. 5. Fracciones de la extracción secuencial para el elemento Fe: I-C: Intercambiable, OX-AM: óxidos amorfos, OXC: óxidos cristalinos, SULF: sulfuros, RES: residual. EPS: entrada presa Santana, IP: inicio presa, MP: mitad presa, CP: cortina presa .

constante. La figura 6 refleja un incremento de las condiciones reductoras que repercuten en el estado de oxidación y las especies que éste formará al ir avanzando hacia la cortina. El porcentaje de hierro asociado a óxidos cristalinos va disminuyendo: $48.2 \%$ para EPS, $43.7 \%$ para IP, $39.1 \%$ para MP y $33.6 \%$ para CP; en cambio, hay un incremento secuencial en la fracción asociada a sulfuros: 28.1 para EPS, $27.6 \%$ para IP, $33.5 \%$ para MP y $42.6 \%$ para CP. Para este elemento, la fracción residual se mantuvo prácticamente constante, lo cual indica la existencia de una fase terrígena que llega mediante transporte físico (arrastre). Las cantidades de hierro total en la superficie del fondo de la presa $(5.08 \mathrm{a}$ $7.98 \%$ ) son mayores que los niveles de 1.2 a $4.6 \%$

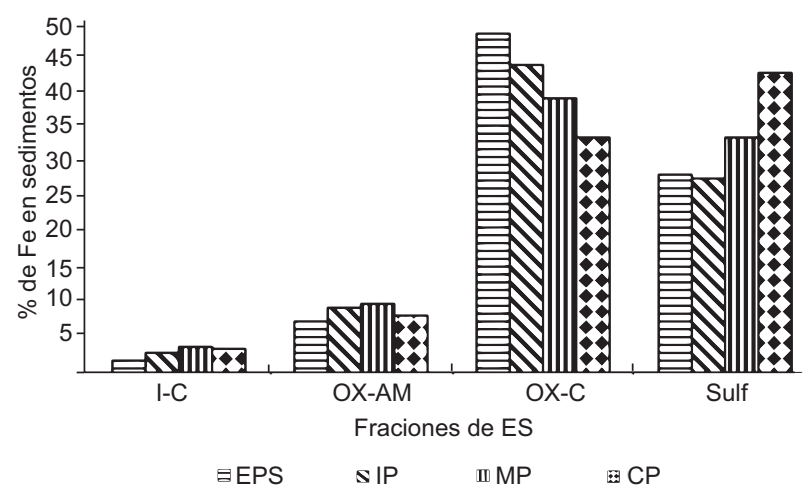

Fig. 6. Contenido porcentual de Fe en las fracciones: I-C: intercambiable, OX-AM: óxidos amorfos, OX-C: óxidos cristalinos, SULF: sulfuros. EPS: entrada presa Santana, IP: inicio presa, MP: mitad presa, CP: cortina presa. de Fe reportados para esta zona por el Servicio Geológico Mexicano (SGM), como refiere la Carta Geoquímica por Fierro Guanajuato F14-C34 (SGM 1998a). Estas cantidades mayores a los niveles de fondo reflejan la entrada de agua de mina y la condición reductora en la cortina, la cual favorece los procesos de disolución reductiva (que libera al ferroso) y de sulfato reducción, que tiene como producto la precipitación de pirita que se va acumulando en el fondo.

El manganeso (Fig. 7) no muestra tendencias claras en cuanto a las fases en que se presenta; sin embargo, el contenido total se encuentra a mayor concentración en los sedimentos cercanos a la cortina $(862.3 \mathrm{mg} / \mathrm{kg})$ que en el resto de los sitios $(562.3$ $\mathrm{mg} / \mathrm{kg}$ ), debido posiblemente a las condiciones reductoras. Los valores basales para la zona en que se encuentra la presa varían de 120 a $900 \mathrm{mg} / \mathrm{kg}$ de acuerdo con la Carta Geoquímica por Manganeso Guanajuato F14-C34 (SGM 1998b).

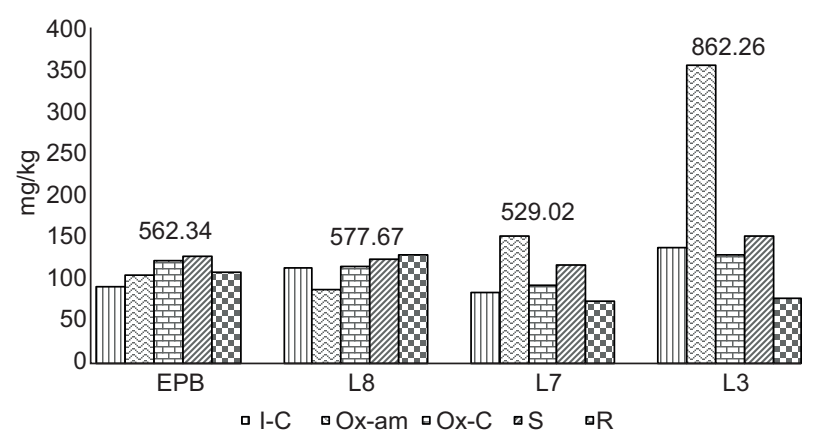

Fig. 7. Fracciones de la extracción secuencial para el elemento Mn: I-C: intercambiable, OX-AM: óxidos amorfos, OX-C: óxidos cristalinos, S: sulfuros, R: residual. EPS: entrada presa Santana, IP: inicio presa, MP: mitad presa, $\mathrm{CP}$ : cortina presa.

El contenido de plomo total (Fig. 8) refleja una acumulación en las zonas cercanas a la cortina (EPS, $0.64 \mathrm{mg} / \mathrm{kg}$; IP, $0.55 \mathrm{mg} / \mathrm{kg}$; MP, $1.66 \mathrm{mg} / \mathrm{kg}$, y CP, $2.83 \mathrm{mg} / \mathrm{kg}$ ). Conforme se avanza en el transecto disminuye la concentración de $\mathrm{Pb}$ en la fracción soluble, a la vez que aumenta su concentración en la fracción de óxidos cristalinos y la fracción residual.

El cuadro IV muestra una comparativa con las concentraciones de $\mathrm{Fe}$, As, Mn y $\mathrm{Pb}$ que se han encontrado en sedimentos en distintos lugares del mundo. Ahí es posible apreciar que los valores son muy variables para cada sitio en función de la geología de cada región y de las actividades de extracción de minerales realizadas. 


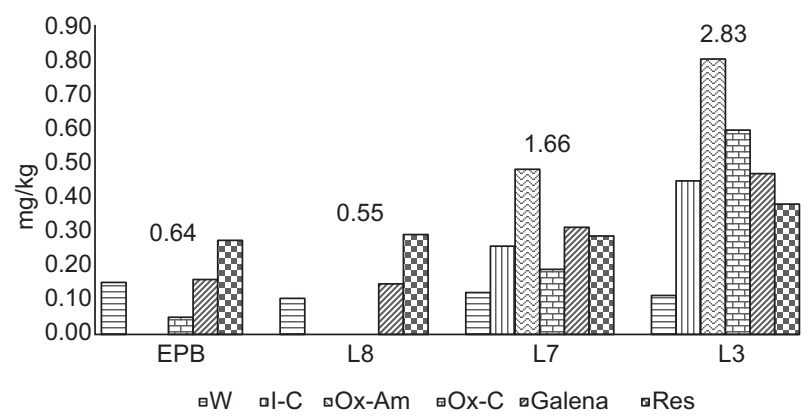

Fig. 8. Fracciones de la extracción secuencial para el elemento $\mathrm{Pb}$ : W: agua, I-C: intercambiable, OX-AM: óxidos amorfos, OX-C: óxidos cristalinos, RES: residual. EPS: entrada presa Santana, IP: inicio presa, MP: mitad presa, $\mathrm{CP}$ : cortina presa.

Los valores de fondo establecidos por el SGM para el porcentaje de $\mathrm{Fe}$ (1.2-4.6) son menores que los reportados en el presente estudio. Para la PS se encontró en un rango de 5.13 a $7.98 \%$, lo cual confirma que el cuerpo de agua está enriqueciéndose en este metal en los sedimentos. Para este mismo metal el mayor valor reportado (Valente et al., 2015) es de $35 \%$ en sedimentos del río Tinto en España, donde se ubica la mayor anomalía natural de pirita en el mundo. Para el As, el mayor valor (12 $109 \mathrm{mg} / \mathrm{kg})$ también fue reportado por Valente et al. (2015), y se debe a las altas cantidades de arsenopirita en los yacimientos de la Faja Pirítica Ibérica. Para este estudio los rangos de As total en los sedimentos de la PS son de 25.11$28.8 \mathrm{mg} / \mathrm{kg}$, que resultan menores a los valores de fondo para sedimentos en la región según los datos del SGM (1998c) (60-120 mg/kg). Estos datos confirman que la PS no actúa como un sumidero de As, y sugieren una movilización física del metaloide asociado a material particulado en eventos de escurrimientos máximos en temporada de lluvias. Los valores de Mn encontrados en este estudio fueron de 562.3 a 862.3 $\mathrm{mg} / \mathrm{kg}$. El SGM reporta valores de fondo para Mn en esta región de 120-900 mg/kg, los cuales están en el rango de los reportados en este estudio. Para $\mathrm{Pb}$, los valores máximos (11 $922 \mathrm{mg} / \mathrm{kg})$ se han reportado también para la región de la Faja Pirítica Ibérica (Valente et al. 2015), mientras que los datos de este estudio fueron de 0.64 a $2.83 \mathrm{mg} / \mathrm{kg}$, valor muy inferior a los de fondo reportados por el SGM de 23-124 mg/kg.

\section{Obtención de micrografías de las muestras de sedimento}

Mediante el uso de esta herramienta se pudo constatar que la presencia de hierro es una constante en las muestras analizadas, y lo que varía es la concentración. En la mayoría de las muestras analizadas se puede encontrar una composición predominante de silicatos. La figura 9 corresponde a una micrografía tomada con el microscopio electrónico de barrido a una muestra de sedimento ubicada en la cortina de la presa. Se puede observar que es calcita sobre la que se formó ferrihidrita. Se observa que ocupa prioritariamente espacios

CUADRO IV. COMPARACIÓN DE LOS VALORES ELEMENTALES BASALES (mg/kg) PARA DISTINTOS SITIOS (As, $\mathrm{Mn}$ y $\mathrm{Pb}$ ).

\begin{tabular}{|c|c|c|c|c|c|}
\hline SITIO & $\begin{array}{l}\mathrm{Fe} \\
(\%)\end{array}$ & As & $\mathrm{Mn}$ & $\mathrm{Pb}$ & Referencia \\
\hline $\begin{array}{l}\text { Castilseras, } \\
\text { Valdeazogues, } \\
\text { España }\end{array}$ & $3.05-5.17$ & $8.32-14.3$ & $433-666$ & $20.9-50.8$ & García- Ordiales et al. 2015 \\
\hline Manwan, China & $1.91-3.79$ & $10.3-72.6$ & $246.6-769.1$ & $17.03-92.2$ & Wang et al. 2012 \\
\hline Hungfeng, China & $1.36-8.57$ & $0.1-49.1$ & ---------------- & $1.2-89.2$ & Wang et al. $2015 \mathrm{a}$ \\
\hline $\begin{array}{l}\text { Dunping Lake, } \\
\text { China }\end{array}$ & ------------- & $19.2-38.5$ & ---------------- & $29.2-41.3$ & Wang et al. $2015 \mathrm{~b}$ \\
\hline Alquera, Portugal & $0.8-22.4$ & $3.66-11.84$ & ----------------- & $0-197.4$ & Palma et al. 2014 \\
\hline Rio Tinto, España & $5.5-35$ & $976-12,109$ & $77-722$ & $61.3-11,922$ & Valente et al. 2015 \\
\hline $\begin{array}{l}\text { Presa Santana } \\
\text { Guanajuato, México }\end{array}$ & $5.13-7.98$ & 25.11-28.8 & $562.3-862.3$ & $0.64-2.83$ & Presente estudio \\
\hline Valores de fondo & $1.2-4.6$ & $60-120$ & $120-900$ & $23-124$ & $\begin{array}{l}\text { Servicio Geológico } \\
\text { Mexicano }\end{array}$ \\
\hline
\end{tabular}




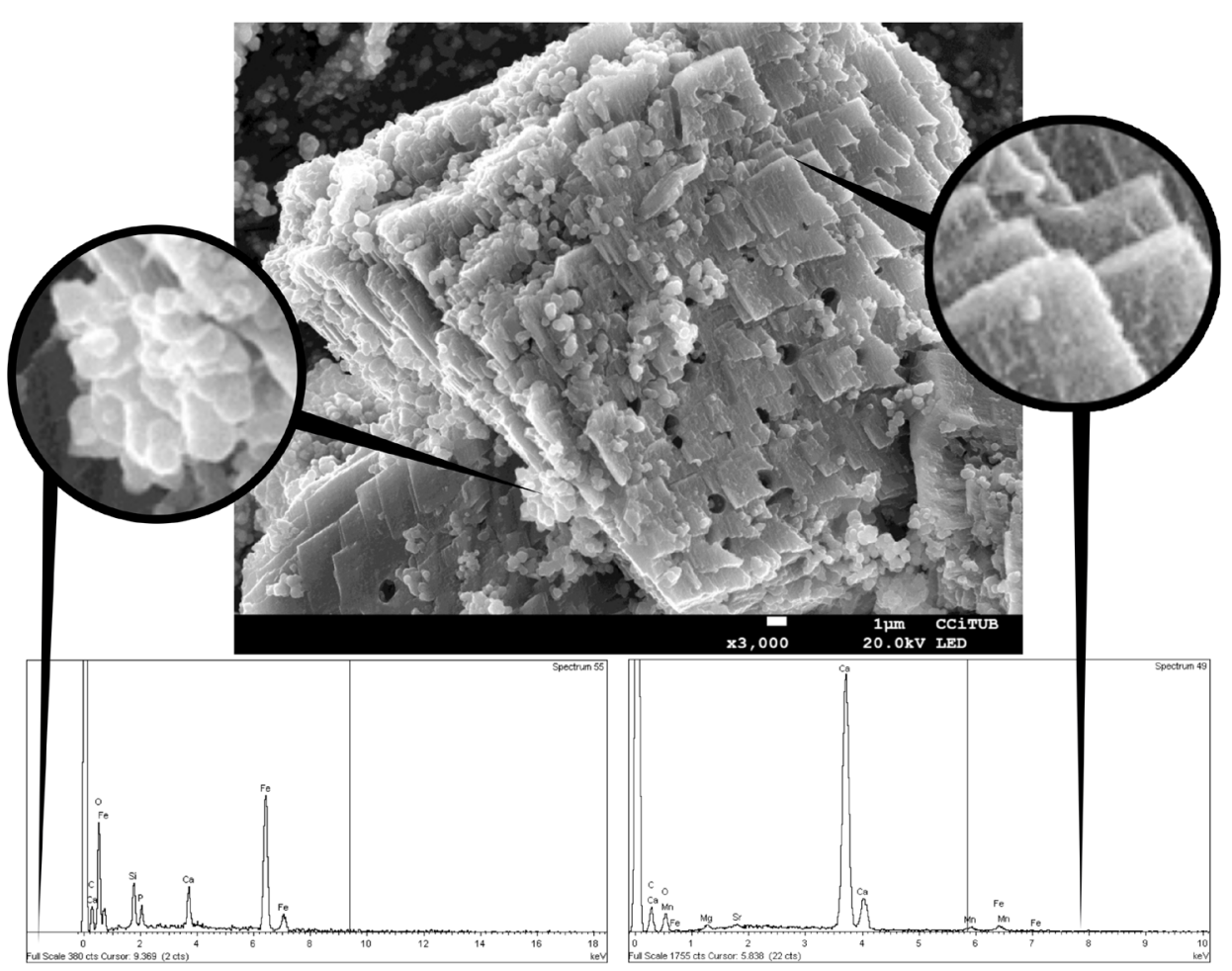

Fig. 9. Muestra de sedimentos donde se aprecian calcita (apariencia cúbica) y ferrihidrita (apariencia esférica).

vacíos y en los bordes del mineral. La figura 10 es un acercamiento específico a la ferrihidrita, donde se aprecia su estructura característica. En la figura 11 se aprecia un mineral con apariencia semejante a un fragmento de pirita, lo cual se corroboró con el análisis mediante detector de energía dispersiva (EDS, por sus

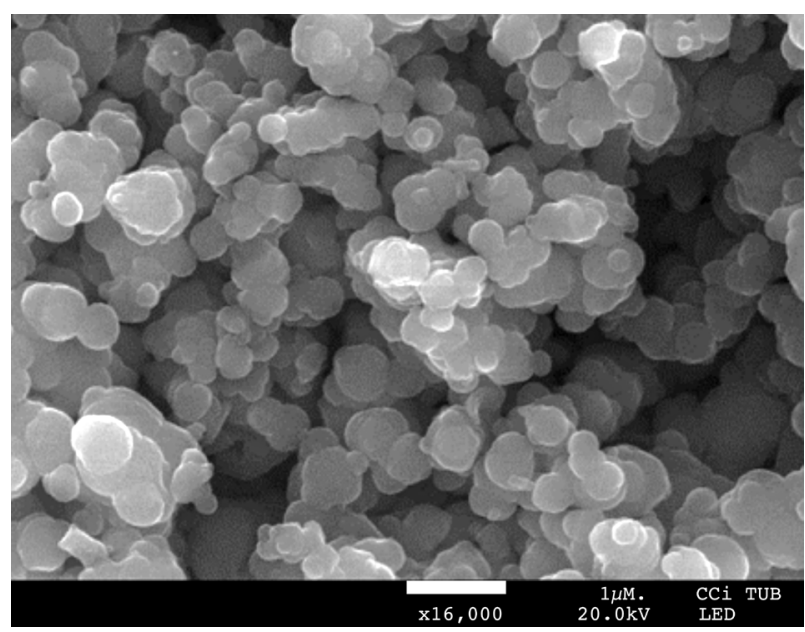

Fig. 10. Ampliación de la micrografía de la muestra de sedimentos donde se aprecia ferrihidrita (apariencia esférica). siglas en inglés), el cual se muestra en la figura 12. Este mismo análisis confirma la presencia de As dentro de la estructura de la pirita, lo que denota que se trata de pirita arsenical (FeS + As). Los filamentos que se observan sobre la pirita son la formación de sulfatos secundarios. De acuerdo con análisis semicuantitativo

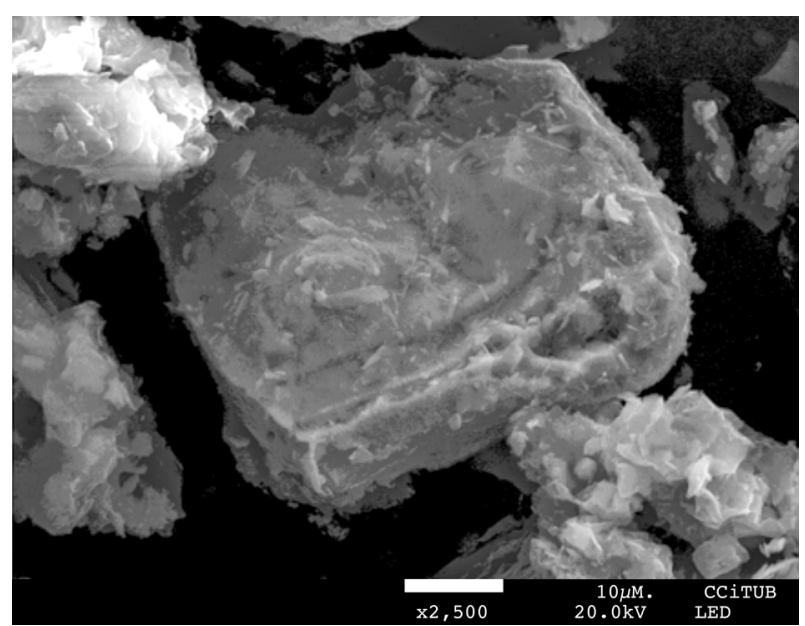

Fig. 11. Pirita arsenical con formación de minerales secundarios sobre este mineral. 


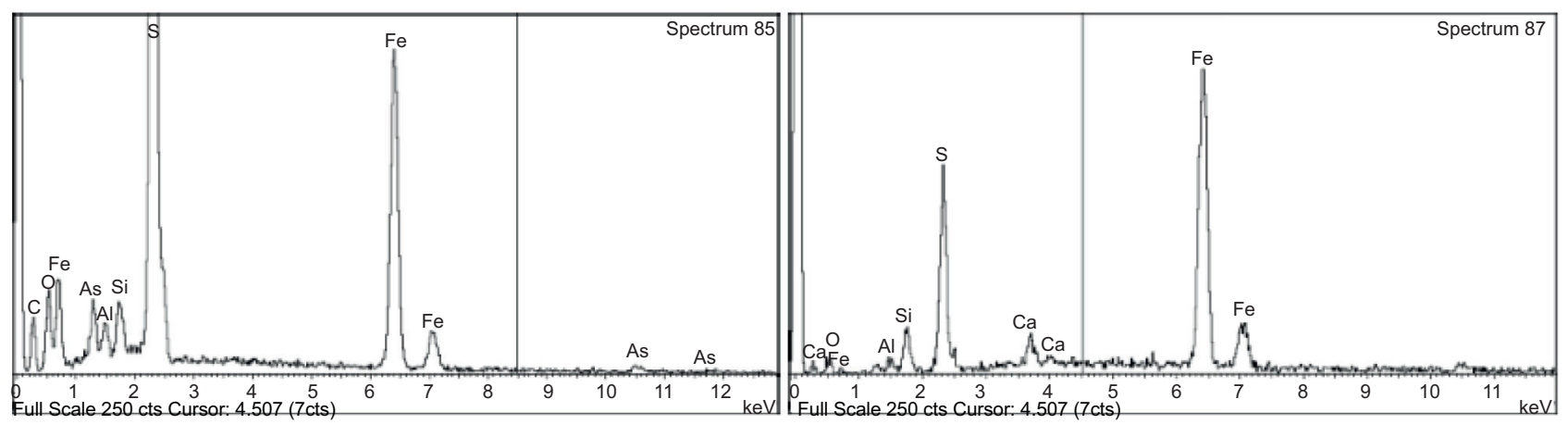

Fig. 12. Elementos presentes en la muestra. Gráficas generadas mediante detector de energía dispersiva.

realizado con el detector EDS, se estableció la existencia de yeso ( $\mathrm{Ca}, \mathrm{S}$ y $\mathrm{O})$ en la segunda grafica (derecha). Se deduce que está ocurriendo una oxidación en la estructura de pirita arsenical, dando lugar a la formación de yeso. Sin embargo, también se encontraron ciertos elementos en la matriz de la muestra $(\mathrm{Cr}, \mathrm{Ti}, \mathrm{Zn}, \mathrm{Fe}$ y $\mathrm{Mn}$ ) conforme se analizó puntualmente en distintos sitios. La presencia de los primeros tres elementos se debe a un posible arrastre de materiales particulados proveniente de los escurrimientos de mina.

\section{CONCLUSIONES}

Se aplicó un protocolo de extracciones secuenciales a cuatro muestras de sedimentos más un duplicado, recolectados en una presa que ha recibido escurrimientos neutros a ligeramente alcalinos y sulfatados de minas epitermales. Los resultados sugieren que existe un transecto geoquímico con una variación de las condiciones de oxidación-reducción (redox) de -0.2 a $0.3 \mathrm{~V}$, y de $\mathrm{pH}$ de 7.46 a 10.02 , controlado por el contenido de materia orgánica, por la condición de ser un cuerpo de agua somero (máximo de $5 \mathrm{~m}$ de profundidad) donde el ambiente reductor es controlado por el consumo de oxígeno. En otros sitios (presas más profundas), el principal factor que controla la condición redox es la difusión de oxígeno. La ubicación (tanto longitudinalmente, a lo largo del eje principal de la presa, como de profundidad) es determinante para las condiciones que influyen en la movilidad de los elementos.

Se aprecia que el As (predominantemente As V) entra en forma particulada, asociado a óxidos de hierro (amorfos y cristalinos). La fase que lo contiene se va transformando a lo largo de la presa, siendo más relevante en la cortina la fracción de sulfuros. La cantidad de As total es casi constante en todas las zonas de la presa, lo que sugiere que, habiendo una transformación en las especies minerales que lo contienen, no se acumula y puede migrar en suspensión durante los episodios de avenidas máximas en temporada de lluvias.

El Fe total tiende a incrementarse en función de la cercanía a la cortina, y al entrar a la presa se encuentra predominantemente en la fracción de los óxidos (amorfos y cristalinos). Es evidente un incremento en la fracción de sulfuros en la cortina, lo cual se ve favorecido por el ambiente reductor, donde hay sulfato reducción y precipitación de sulfuros ferrosos. Este parámetro sobrepasa lo establecido por el SGM para los valores de fondo (1.2-4.6\% para Fe), por lo que es claro que la presa actúa como depósito de hierro.

Para el Mn, la concentración total es prácticamente la misma en las tres primeras muestras; sin embargo, en la muestra próxima a la cortina el valor aumenta significativamente (556-862.26 mg/kg).

Las información aportada por micrografías obtenidas mediante SEM confirma el contenido elemental encontrado en las tendencias de las extracciones secuenciales. Se observó la presencia abundante de óxidos de hierro y manganeso en las muestras.

\section{AGRADECIMIENTOS}

Al personal del Laboratorio de Hidrología del Consejo Superior de Investigaciones CientíficasInstituto de Diagnóstico Ambiental y Estudios del Agua (CSIC-IDAEA), Barcelona, España, en especial a Jordi Bellés. Al Grupo de Trabajo de Hidrogeoquímica de la Universidad de Guanajuato. Al Dr. Raúl Miranda por las atenciones brindadas. A la División de Ingenierías del Consejo Nacional de Ciencia y Tecnología (CONACYT) por el apoyo a esta investigación. Y a los amables revisores anónimos que enriquecieron este trabajo. 


\section{REFERENCIAS}

Aguilar-Hinojosa Y., Meza-Figueroa D., Villalba-Atondo A.I., Encinas-Romero M.A., Valenzuela-García J.L. y Gómez-Álvarez A. (2016). Mobility and bioavailability of metals in stream sediments impacted by mining activities: The Jaralito and the Mexicana in Sonora, Mexico. Water Air Soil Pollut. 227 (9), 345. https:// doi.org/10.1007/s11270-016-3046-1

Armiento G., Nardi E., Lucci F., De Cassan M., Della Ventura G., Santini C., Petrini E. y Cremisini C. (2017). Antimony and arsenic distribution in a catchment affected by past mining activities: Influence of extreme weather event. Water Air Soil Poll. 28 (2), 303-315. https://doi.org/10.1007/s12210-016-0566-y

Bravo-Covarrubias A., Serafín A.H., Cano-Rodríguez I., Gutiérrez-Valtierra M., Vera R., Jeanneau E. y RamosArroyo Y.R. (2015). Spatial and temporal variability of the chemistry of a dam that receives mine drainage. Memorias. Solución a la problemática del agua en México: propuestas de jóvenes investigadores. Guanajuato, México, 27 al 29 abril. CD-ROM.

Bravo-Covarrubias A., Cano-Rodríguez I., Jofre-Meléndez R., Gutiérrez-Valtierra M. y Ramos-Arroyo Y.R. (2017). Mass balances in a dam that receives mine drainage. Procedia Earth and Planet. Sci. 17, 710-713. https://doi.org/10.1016/j.proeps.2016.12.167

Bowell R.J. (1994). Sorption of arsenic by iron oxides and oxyhydroxides in soils. Appl. Geochem. 9 (3), 279286. https://doi.org/10.1016/0883-2927(94)90038-8

CCME (2014). Canadian sediment quality guidelines for the protection of aquatic life. Canadian Council of Ministers of the Environment [en línea]. http://ceqgrcqe.ccme.ca/download/en/226/29/08/2019

Camprubí A. y Albinson T. (2006). Depósitos epitermales en México: actualización de su conocimiento y reclasificación empírica. B. Soc. Geol. Mex. 58 (1), 27-81. https://doi.org/10.18268/bsgm2006v58n1a2

Chabukdhara M. y Nema A.K. (2012). Assessment of heavy metal contamination in Hindon River sediments: A chemometric and geochemical approach. Chemosphere 87 (8), 945-953. https://doi.org/10.1016/j. chemosphere.2012.01.055

Dold B. (2003). Speciation of the most soluble phases in a sequential extraction procedure adapted for geochemical studies of copper sulfide mine waste. J. Geochem. Explor. 80 (1), 55-68. https://doi.org/10.1016/S03756742(03)00182-1

DWR (1995). Compilation of sediment and soil standards, criteria and guidelines. Department of water resources, state of California [en línea]. https://www. water.ca.gov/LegacyFiles/pubs/waterquality/municipal_wq_investigations/mwqi_technical_documents/ compilation_of_soil_and_sediment_standards_criteria_and_guidelines/compilation_of_soil_and_sediment_standards_criteria_and_guidelines._february_1995.pdf 24/05/2018

Dzombak D.A. y Morel M.M. (1990). Surface complexation modeling: Hydrous ferric oxide. John Wiley and Sons, Nueva York, EUA, 393 pp.

Frémion F., Bordas F., Mourier B., Lenain J.F., Kestens T. y Courtin-Nomade A. (2016). Influence of dams on sediment continuity: A study case of a natural metallic contamination. Sci. Total Environ. 547 (3), 282-294. https://doi.org/10.1016/j.scitotenv.2016.01.023

García-Ordiales E., Esbrí J.M., Covelli S., López-Berdonces M.A., Higueras P.L. y Loredo J. (2016). Heavy metal contamination in sediments of an artificial reservoir impacted by long-term mining activity in the Almadén mercury district (Spain). Environ. Sci. Pollut. Res. 23 (7), 6024-038. https://doi.org/10.1007/s11356-015-4770-6

Hansen A.M. (2012). Lake sediment cores as indicators of historical metal(oid) accumulation - A case study in Mexico. Appl. Geochem. 27 (9), 1745-1752. https:// doi.org/10.1016/j.apgeochem.2012.02.010

Jofre-Meléndez R., Ramos-Arroyo Y. y Botello-Rionda S. (2017). Orographic shading effect on water/sediment heat exchange in two dams of Guanajuato river, Mexico. Proced. Earth Planet. Sci. 17 (1), 873-876. https://doi.org/10.1016/j.proeps.2017.01.002

Kim E., Yoo J. y Baek K. (2014). Arsenic speciation and bioaccessibility in arsenic contaminated soils: Sequential extraction and mineralogical investigation. Environ. Pollut. 186 (3), 29-35. https://doi.org/10.1016/j. envpol.2013.11.032

Lee K., Shim H., Lee D. y Chung D. (2015). The fate and factors determining arsenic mobility of arsenic in soil - A review. Korean J. Soil Sci. Fert. 48 (2), 73-80. https://doi.org/10.7745/KJSSF.2015.48.2.073

Martínez-Arredondo J.C., Jofre Meléndez R., Ortega Chávez V.M. y Ramos Arroyo Y.R. (2015). Descripción de la variabilidad climática normal (1951-2010) en la cuenca del río Guanajuato, centro de México. Acta Universitaria 25 (6), 31-47. https://doi.org/10.15174/ au.2015.799

Maull E.A., Ahsan H., Edwards J., Longnecker M.P., Navas A., Pi J., Silbergeld E.K., Styblo M., Tseng C.H. y Thayer K.A. (2012). Evaluation of the association between arsenic and diabetes: A National Toxicology Program workshop review. Environ. Health Perspect. 120 (12), 1658-1670. https://doi.org/10.1289/ ehp. 1104579

Moon K., Guallar E. y Navas-Acien A. (2012). Arsenic exposure and cardiovascular disease: An update systematic review. Curr. Atheroscler. Rep. 14 (6), 542-555. https://doi.org/10.1007/s11883-012-0280-x 
Nordstrom D.K. (2011). Hydrogeochemical processes governing the origin, transport and fate of major and trace elements from mine wastes and mineralized rock to surface waters. Appl. Geochem. 26 (11), 1777-1791. https://doi.org/10.1016/j.apgeochem.2011.06.002

Palma P., Ledo L., Soares S., Barbosa I.R. y Alvarenga P. (2014). Spatial and temporal variability of the water and sediments quality in the Alqueva reservoir (Guadiana Basin; southern Portugal). Sci. Total Environ. 470-471 (3), 780-790. https://doi.org/10.1016/j.scitotenv.2013.10.035

Parkhurst D.L. y Appelo C.A.J. (2013). Description of input and examples for PHREEQC version 3--A computer program for speciation, batch-reaction, one-dimensional transport, and inverse geochemical calculations [en línea]. https://pubs.usgs.gov/tm/06/a43/ 21/05/2018

Plumlee G.S. (1999). The environmental geology of mineral deposits. En: Reviews in economic geology, vol. 6A. (Plumlee G.S. y Logsdon M.J., Eds.). Society of Economic Geologists, Inc. Federal Center, Denver, EUA, pp. 71-116.

Puigdomenech I., Colas E., Grive M., Campos I. y García D. (2014). A tool to draw chemical equilibrium diagrams using SIT: Applications to geochemical systems and radionuclide solubility. Mater. Res. Soc. Symp. Proc. 1665 (1), 111-116. DOl: 10.1557/opl.2014.635

Ramos Y.R., Muñoz D., Rodríguez I., Jiménez G., Ramírez V. y Martínez J.C. (2010). Detrimental effects of mining on water quality in a small catchment of Guanajuato, México. Proceedings of the 13th International Conference on Water-Rock Interaction. Guanajuato, Guanajuato, México. 16 al 20 agosto. CD-ROM.

Randall J.A., Saldana E., Clark K.F. (1994). Exploration in a volcano-Plutonic center at Guanajuato, Mexico. Econ. Geol. 89 (8), 1722-1751. https://doi.org/10.2113/ gsecongeo.89.8.1722

Rao C.R.M., Sahuquillo A. y López J.F. (2008). A review of the different methods applied in environmental geochemistry for single and sequential extraction of trace elements in soils and related materials. Water Air Soil Pollut. 189 (1-4), 291-333. https://doi.org/10.1007/ s11270-007-9564-0

Rauret G., López J.F., Sahunquillo A., Rubio R., Davidson C.I. y Ure A. (1999). Improvement of the BCR three step sequential extraction procedure prior to the certification of the new sediment and soil reference materials. J. Environ Monit. 1 (1), 54-61. https://doi. org/10.1039/A807854H

Romero F.M., Armienta M.A. y Carrillo R. (2004). Arsenic sorption by carbonate-rich aquifer material, a control on arsenic mobility at Zimapán, México. Arch. Environ. Contam. Toxicol. 47 (1), 1-13. https://doi. org/10.1007/s00244-004-3009-1
SGM (1998a). Carta geoquímica por fierro Guanajuato F14-C43. Servicio Geológico Mexicano [en línea]. https://mapserver.sgm.gob.mx/Cartas Online/geoquimica/1416_F14-C43 fe.pdf 24/05/2018

SGM (1998b). Carta geoquímica por manganeso Guanajuato F14-C43. Servicio Geológico Mexicano [en línea]. https://mapserver.sgm.gob.mx/Cartas_Online/ geoquimica/1416_F14-C43_mn.pdf 24/05/2018

SGM (1998c). Carta geoquímica por arsénico Guanajuato F14-C43. Servicio Geológico Mexicano [en línea]. https://mapserver.sgm.gob.mx/Cartas_Online/geoquimica/1416 F14-C43 as.pdf 24/05/2018

Sharifi R., Moore F. y Keshavararzi B. (2016). Mobility and chemical fate of arsenic and antimony in water and sediments of Sarouq River catchment, Takab geothermal field northwest Iran. J. Environ. Manag. 170 (6), 136144. https://doi.org/10.1016/j.jenvman.2016.01.018

Smedley P.L. y Kinniburgh D.G. (2002). A review of the source, behaviour and distribution of arsenic in natural waters. Appl. Geochem. 17 (5), 517-568. https://doi. org/10.1016/S0883-2927(02)00018-5

Smedley P.L., Kinniburgh D.G., Macdonald D.M.J., Nicolli H.B., Barros A.J., Tullio J.O., Pearce J.M. y Alonso M.S. (2005). Arsenic associations in sediments from the loess aquifer of La Pampa, Argentina. Appl. Geochem. 20 (5), 989-1016. https://doi.org/10.1016/j. apgeochem.2004.10.005http://dx.doi.org/10.1016/j. apgeochem.2004.10.005

SSA (1994). Norma Oficial Mexicana NOM-127SSA1-1994. Salud ambiental, agua para uso y consumo humano-límites permisibles de calidad y tratamientos a que debe someterse el agua para su potabilización. Secretaría de Salud. Diario Oficial de la Federación. 30 de noviembre.

Tessier A., Campbell P.G.C. y Bisson M. (1979). Sequential extraction procedure for speciation of particulate trace metals. Anal. Chem. 51 (7), 844-851. https://doi. org/10.1021/ac50043a017

Torres E. y Auleda M. (2013). A sequential extraction procedure for sediments affected by mine drainage. J. Geochem. Expl. 128 (4), 35-41. https://doi. org/10.1016/j.gexplo.2013.01.012

Valente T., Grande J.A., de la Torre M.L., Gomes P., Santisteban M., Borrego J. y Sequeira-Braga M.A. (2015). Mineralogy and geochemistry of a clogged mining reservoir affected by historical acid mine drainage in an abandoned mining area. J. Geochem. Explor. 157 (10), 66-76. https://doi.org/10.1016/j.gexplo.2015.05.016

Varol M. (2013). Dissolved heavy metal concentrations of the Kralkizi, Dicle and Batman dam reservoirs in the Tigris River basin, Turkey. Chemosphere 93 (6), 954-962. https://doi.org/10.1016/j.chemosphere.2013.05.061 
Wan X., Don H., Feng L., Lin Z. y Luo Q. (2017). Comparison of three sequential extraction procedures for arsenic fractionation in highly polluted sites. Chemosphere 178, 402-410. https://doi.org/10.1016/j. chemosphere.2017.03.078

Wang C., Liu S., Zhao Q., Deng L. y Dong S. (2012). Spatial variation and contamination assessment of heavy metals in sediments in the Manwan Reservoir, Lancang River. Ecotox. Environ. Safe 82 (8), 32-39. https://doi.org/10.1016/j.ecoenv.2012.05.006
Wang G., Yinglan A., Jiang H., Fu Q. y Zheng B. (2015a). Modeling the source contribution of heavy metals in surficial sediment and analysis of their historical changes in the vertical sediments of a drinking water reservoir. J. Hydrol. 520 (1), 37-51. https://doi. org/10.1016/j.jhydrol.2014.11.034

Wang Y., Yang L., Kong L., Liu E., Wang L. y Zhu J. (2015b). Spatial distribution, ecological risk assessment and source identification for heavy metals in surface sediments from Dongping Lake, Shandong, East China. Catena 125 (2), 200-205. https://doi. org/10.1016/j.catena.2014.10.023 